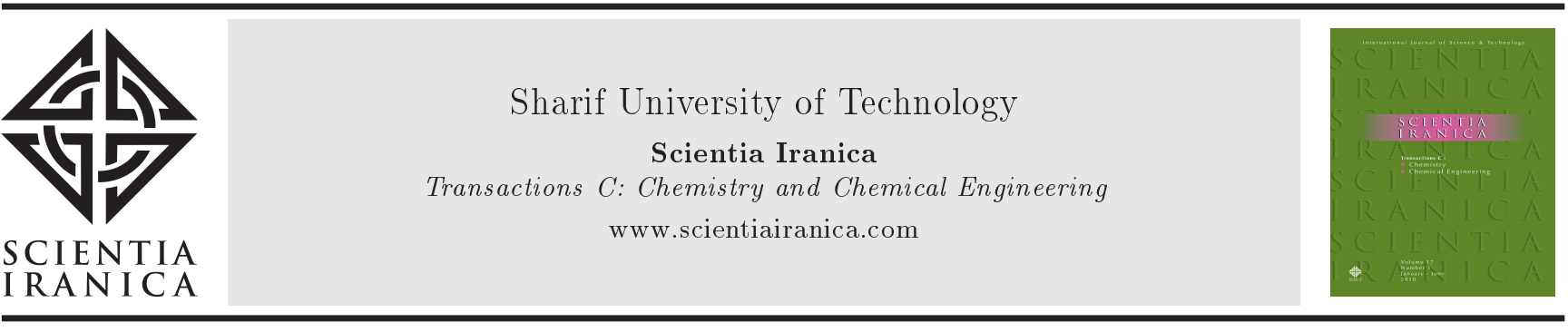

\title{
Application of conformal mapping to the scattering of plane SH-waves by a cavity
}

\author{
W. Chen ${ }^{1, *}$ and S.X. Wang \\ CNPC Key lab of Geophysical Exploration, China University of Petroleum, Beijing, China 102249.
}

Received 23 November 2014; received in revised form 20 August 2015; accepted 24 May 2016

\section{KEYWORDS}

Conformal mapping;

Cavity;

Scattering;

Seismic wave;

Numerical modelling.

\begin{abstract}
In seismic numerical modelling, it is readily to simulate the wave field of the cavities with simple and regular geometric boundaries. However, the real cavities are always complex or irregular, such as general quadrilateral or horny model. In this paper, conformal mapping is applied to three representative cavity models, including a pentagon model, a generalized quadrangular model, and a horny model. First of all, we transform the original cavity model in the physical domain into a certain simple regular model in the computational domain and, accordingly, transform the boundary condition in the physical domain into that in the computational domain. Then, the wave field on the boundary in the computational domain is calculated. Finally, we generate the wave field on the boundary in the physical domain by using the inverse conformal mapping, when the conformal mapping function is invertible. Two experiments by adopting either a displacement boundary condition or a stress boundary condition illustrate that the wave fields for the three different kinds of cavities mainly concentrate on the boundary of the corresponding cavity.
\end{abstract}

(C) 2016 Sharif University of Technology. All rights reserved.

\section{Introduction}

In exploration geophysics discipline, some cavities in subsurface rocks are often related to reservoirs, since they can load oil or gas. Therefore, it is important to analyze the response character of seismic wave by a cavity $[1,2]$. It is easy to simulate the wave field of the cavities with simple and regular geometric boundaries by using numerical methods or even analytical methods. However, it is difficult to directly simulate a complex or irregular model, which represents real cavity model.

The conformal mapping [3] provides an idea which transforms complex or irregular model in the original

1. Present addresses: Key Laboratory of Exploration Technology for Oil and Gas Resources of Ministry of Education, Yangtze University, Wuhan Hubei 430100; and 2 Hubei Cooperative Innovation Center of Unconventional Oil and Gas, Wuhan Hubei 430100.

*. Corresponding author.

E-mail addresses: waqw242@sina.com (W. Chen); 284059127r@qq.com (S.X.Wang) (physical) domain into simple and regular model in the computational domain. In this way, complex or irregular model can be indirectly simulated. In nature, the conformal mapping or some similar techniques, which transform complex or unsolvable or ill-posed problems in the original domain into simple or solvable or well-posed problems in a certain transform domain, have been studied in seismic wave modelling [4,5], seismic processing [6,7], and seismic inversion [8]. In this paper, we apply the conformal mapping to the regular pentagon model, the generalized quadrangular model and the horny model, and analytically derive and numerically simulate the wave field with either a displacement boundary condition or a stress boundary condition.

\section{Theory}

\subsection{The scattering wave field}

Consider a 3-D isotropic elastic medium embedded into an infinitely long cylindrical cavity (Figure 1). The 


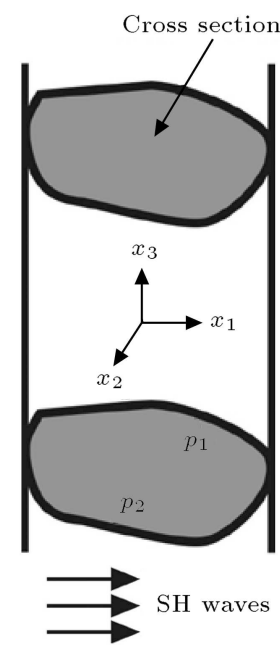

Figure 1. The cavity model and its boundary $p=p_{1}+p_{2}$. The shaded part in the model is the cross section of the cavity. Cross section is on the $x_{1} x_{2}$ plane and the cavity stretches along $x_{3}$ axis.

elastodynamic equations without body forces can be described as [9]:

$$
\begin{aligned}
& \sigma_{l m}=\lambda u_{k, k} \delta_{l m}+2 \mu \varepsilon_{l m}, \\
& \sigma_{m l, m}=\rho \frac{\partial^{2} u_{l}}{\partial t^{2}}, \\
& \varepsilon_{l m}=\left(u_{l, m}+u_{m, l}\right) / 2,
\end{aligned}
$$

where $\sigma_{l m}$ is the stress, $u$ is the displacement, $\delta(\bullet)$ is Dirac-Delta function, $\varepsilon_{l m}$ is the strain, and $\lambda$ and $\mu$ are the Lame constants. The subscripts $l, m$, and $k$ denote three spatial variables. A comma after a quantity denotes partial derivative with respect to spatial variable. For example, $u_{l, m}$ denotes partial derivative of the displacement along the $l$ direction with respect to spatial variable $m$.

Substitution of Eq. (1) into Eq. (2) yields the wellknown Navier-Cauchy equation:

$$
\left(c_{l m p q} u_{p, q}\right)_{, m}=\rho \frac{\partial^{2} u_{l}}{\partial t^{2}},
$$

where $c_{l m p q}$ is the fourth-order elasticity tensor. The traditional summation rule over repeated indices is applied throughout this paper.

We consider the scattering problem of plane SHwaves by three kinds of infinitely long cylinder cavities with different cross sections in this paper. Assuming the cross section is on the $x_{1} x_{2}$ plane and the cavity stretches along $x_{3}$ axis (Figure 1 ), the $3-\mathrm{D}$ problem can be expressed by a 2-D problem. That means:

$$
\begin{aligned}
& u_{1}=u_{2}=0, \\
& u_{3}=u_{3}\left(x_{1}, x_{2}, t\right) .
\end{aligned}
$$

By substituting Eqs. (5) and (6) into Eq. (2), we obtain:

$$
v_{s}^{2} \nabla^{2} u_{3}=\frac{\partial^{2} u_{3}}{\partial t^{2}}
$$

where $v_{s}=(\mu / \rho)^{1 / 2}$ is the shear wave velocity, and $\nabla^{2}=\frac{\partial^{2}}{\partial x_{1}^{2}}+\frac{\partial^{2}}{\partial x_{2}^{2}}$ is a Laplace operator.

In the case of steady state, Eq. (7) becomes a Helmholtz equation:

$$
\nabla^{2} u_{3}+\beta^{2} u_{3}=0
$$

where $\beta=\left(\omega / v_{s}\right)^{1 / 2}$ is the shear wave number.

From Eqs. (1) and (5), we have:

$$
\sigma_{11}=\sigma_{22}=\sigma_{33}=\sigma_{12}=0 \text {, }
$$

and:

$$
\sigma_{3 l}=\mu u_{3, l},
$$

where $l$ is 1 or 2 .

For illustrative purposes, complex variable $p=$ $x_{1}+i x_{2}$ and its complex conjugate $\bar{p}=x_{1}-i x_{2}$ in the original physical domain (also called $p$ domain) are introduce. In this case, Eqs. (8) and (10) can be rewritten as:

$$
4 \frac{\partial^{2} u_{3}}{\partial p \partial \bar{p}}+\beta^{2} u_{3}=0
$$

and:

$$
\begin{aligned}
\tau_{13} & =\mu\left(\frac{\partial u_{3}}{\partial p}+\frac{\partial u_{3}}{\partial \bar{p}}\right), \\
\tau_{23} & =\mu i\left(\frac{\partial u_{3}}{\partial p}+\frac{\partial u_{3}}{\partial \bar{p}}\right) .
\end{aligned}
$$

Note that the shear modulus $\sigma_{l m}(l \neq m)$ is replaced by symbol $\tau_{l m}(l \neq m)$.

According to Eq. (11), the scattering wave field $w^{(s)}$ from the cavity meets:

$$
4 \frac{\partial^{2} w^{(s)}}{\partial p \partial \bar{p}}+\beta^{2} w^{(s)}=0 .
$$

By substituting a conformal mapping $p=F(c)$ into Eq. (13), we obtain the corresponding equation in the computational domain (also called $c$ domain):

$$
\frac{\partial^{2} w^{(s)}}{\partial c \partial \bar{c}}=-\frac{\beta^{2}}{4} \frac{d F}{d c} \frac{d \bar{F}}{d \bar{c}} w^{(s)}
$$

where $c$ is a point in the computational domain.

After separating the variables $c$ and $\bar{c}, w^{(s)}(c, \bar{c})$ can be written as:

$$
w^{(s)}=w_{1}(c) w_{2}(\bar{c})
$$

By substituting Eq. (15) into Eq. (14) and performing 
integrate operator, we have:

$$
\begin{aligned}
& w_{1}(c)=g_{1}(\tau) \exp [i \beta \sigma F(c) / 2], \\
& w_{2}(\bar{c})=g_{2}(\tau) \exp [i \beta \bar{F}(\bar{c}) /(2 \sigma)],
\end{aligned}
$$

where $g_{1}$ and $g_{2}$ are two constant functions determined by a separation constant $\tau$.

Substituting Eq. (16) into Eq. (15) and integrating $\tau$, the general expression of the scattering wave field can be given as:

$$
w^{(s)}=\int_{L} g(\tau) \exp \left\{i \frac{\beta}{2}\left[\tau F(c)+\frac{\bar{F}(\bar{c})}{\tau}\right]\right\} d \tau,
$$

where $L$ is a path on the $c$ domain, which is readily chosen to ensure that the integration in Eq. (17) is convergent.

By setting $\tau=\exp \left(-i \tau_{1}\right)$ and $F(c)=|F(c)| \exp$ $(i \Omega)$, Eq. (17) can be rewritten as:

$$
\begin{aligned}
w^{(s)}= & \int_{L}-i \exp \left(-i \tau_{1}\right) g\left[\exp \left(-i \tau_{1}\right)\right] \exp [i \beta|F(c)| \\
& \left.\cos \left(\tau_{1}-\Omega\right)\right] d \tau_{1}=\int_{L} g_{1}[\exp (-i \tau)] \\
& \exp \left[i \beta|F(c)| \cos \left(\tau_{1}-\Omega\right)\right] d \tau_{1} .
\end{aligned}
$$

The function $g_{1}\left[\exp \left(-i \tau_{1}\right)\right]$ in Eq. (18) can be spread into an infinite series:

$$
g_{1}\left[\exp \left(-i \tau_{1}\right)\right]=\sum_{n=-\infty}^{\infty} a_{n} e^{i n \tau_{1}}
$$

where $a_{n}$ is the coefficients of the series. Introducing a variable $\tau_{2}=\tau_{1}-\Omega-\pi / 2$, Eq. (18) becomes:

$$
\begin{aligned}
w^{(s)}= & \sum_{n=-\infty}^{\infty} a_{n} \exp [\operatorname{in}(\Omega-\pi / 2)] \int_{L} \exp \left(i n \tau_{2}\right. \\
& \left.-i \beta|F(c)| \sin \tau_{2}\right) d \tau_{2} .
\end{aligned}
$$

The result of the integration in Eq. (20) is just a Hankel function $\pi H_{n}^{(1),(2)}[\beta|F(c)|]$.

When $|F(c)| \rightarrow \infty$ :

$$
\begin{gathered}
\pi H_{n}^{(1),(2)}[\beta|F(c)|] \approx \sqrt{\frac{2}{\pi \beta|F(c)|}} \exp \{ \pm i[\beta|F(c)| \\
\left.\left.-\frac{n \pi}{2}-\frac{\pi}{4}\right]\right\}
\end{gathered}
$$

where $H_{n}^{(1),(2)}(\bullet)$ denotes the first kind and the second kind of Hankel function for the $n$th order, respectively.
The scattering wave field in Eq. (20) should meet the following Sommerfeld radiation condition:

$$
\begin{aligned}
& \lim _{c \rightarrow \infty} \sqrt{c}\left|w^{(s)}\right|<M, \\
& \lim _{c \rightarrow \infty} \sqrt{c}\left(\frac{\partial w^{(s)}}{\partial c}-i \beta w^{(s)}\right)=0,
\end{aligned}
$$

where $M$ is a finite constant. Thus, we can only choose the first kind of Hankel function, $H_{n}^{(1)}[\beta|F(c)|]$. Consequently, the final general form of scattering wave field in the computation domain can be expressed as:

$$
w^{(s)}=\sum_{n=-\infty}^{\infty} A_{n} H_{n}^{(1)}(\beta|F(c)|)\left(\frac{F(c)}{|F(c)|}\right)^{n},
$$

where $A_{n}$ is an unknown constant, which requires to be determined from the boundary condition.

\subsection{Boundary conditions}

In order to calculate the scattering wave field by Eq. (24), we must firstly get $A_{n}$. The boundary conditions, such as displacement boundary or stress boundary, need be adopted to solve $A_{n}$.

According to Eq. (12), we have:

$$
\tau_{13}+i \tau_{23}=2 \mu \frac{\partial w}{\partial \bar{p}} .
$$

To conveniently deal with boundary conditions and boundary value relationship, we bring in curvilinear coordinate system $\left(\xi, \eta, x_{3}\right)$. Assuming that the intersection angle of $\xi$ axis and $x_{1}$ axis is $\varphi$, the two shear stress components in the curvilinear coordinate system become:

$$
\begin{aligned}
\tau_{\xi x_{3}}= & \tau_{13} \cos \varphi+\tau_{23} \sin \varphi=\mu\left[\frac{\partial w}{\partial p} \exp (i \varphi)\right. \\
& \left.+\frac{\partial w}{\partial \bar{p}} \exp (-i \varphi)\right], \\
\tau_{\eta x_{3}}= & -\tau_{13} \sin \varphi+\tau_{23} \cos \varphi=\mu i\left[\frac{\partial w}{\partial p} \exp (i \varphi)\right. \\
& \left.-\frac{\partial w}{\partial \bar{p}} \exp (-i \varphi)\right] .
\end{aligned}
$$

Consider that the displacement on boundary $p_{1}$ and the shear stress on boundary $p_{2}$ are known; the boundary condition of the steady-state problem can be expressed as:

$$
\begin{aligned}
& w=f_{1}(p) \quad \forall p \in p_{1} \\
& \mu\left[\frac{\partial w}{\partial p} \exp (i \varphi)+\frac{\partial w}{\partial \bar{p}} \exp (-i \varphi)\right]=f_{2}(p) \quad \forall p \in p_{2}
\end{aligned}
$$

where $f_{1}(p)$ is a known displacement boundary func- 
tion, and $f_{2}(p)$ is a known stress boundary function. Eqs. (27) and (28) are the displacement boundary condition and the stress boundary condition in $p$ domain, respectively. We will introduce the two boundary conditions in $c$ domain to solve $A_{n}$ below.

\subsubsection{Displacement boundary condition}

According to Eqs. (24), (25), and (27), we obtain:

$$
\sum_{n=-\infty}^{\infty} A_{n} H_{n}^{(1)}(\beta|F|)(F /|F|)^{n}=f_{1}-w^{(i)},
$$

where $w^{(i)}$ is the incident wave field. In the case of the polar coordinate $c=\exp (i \theta)$, Eq. (30) can be regarded as a function related to angle $\theta$. To solve $A_{n}$, a term $\exp (-i z \theta)(z=0, \pm 1, \pm 2, \ldots)$ is multiplied by both sides of Eq. (30). Then, it is integrated from $-\pi$ to $\pi$. Subsequently, $A_{n}$ can be calculated by solving the following equation:

$$
\begin{gathered}
\sum_{n=-\infty}^{\infty}\left\{\frac{1}{2 \pi} \int_{-\pi}^{\pi}[L(\theta) / \mid L(\theta)]^{n} H_{n}^{(1)}[\beta \mid L(\theta)]\right. \\
\exp (-i z \theta) d \theta\} A_{n}=\frac{1}{2 \pi} \int_{-\pi}^{\pi}\left[f_{1}(\theta)\right. \\
\left.-w^{(i)}(\theta)\right] \exp (-i z \theta) d \theta
\end{gathered}
$$

where $L(\theta)=F\left(e^{i \theta}\right)$.

\subsubsection{Stress boundary condition}

According to the chain rule, we have:

$$
\begin{aligned}
& \frac{\partial w}{\partial p}=\frac{1}{F^{\prime}} \frac{\partial w}{\partial c}, \\
& \frac{\partial w}{\partial \bar{p}}=\frac{1}{\bar{F}^{\prime}} \frac{\partial w}{\partial \bar{c}},
\end{aligned}
$$

where $F^{\prime}=\frac{d F}{d c}, \bar{F}^{\prime}=\frac{d \bar{F}}{d \bar{c}}$.

By substituting Eq. (31) into Eq. (28), we obtain the stress boundary condition in $c$ domain as:

$$
\frac{\mu}{\left|F^{\prime}\right|}\left(c \frac{\partial w}{\partial c}+\bar{c} \frac{\partial w}{\partial \bar{c}}\right)=f_{2} .
$$

Then, we substitute Eq. (32) into Eq. (24) and obtain:

$$
\begin{aligned}
\sum_{n=-\infty}^{\infty} & {\left[c F^{\prime} H_{n-1}(\beta \mid c)(F /|F|)^{n-1} \overline{c F^{\prime}} H_{n+1}(\beta \mid F)\right.} \\
& \left.(F /|F|)^{n+1}\right] A_{n}=\frac{2}{\beta}\left[\frac{\left|F^{\prime}\right|}{\mu} f_{2}-c \frac{\partial w^{(i)}}{\partial c}\right. \\
& \left.-\bar{c} \frac{\partial w^{(i)}}{\partial \bar{c}}\right] .
\end{aligned}
$$

Similar to the steps for deriving Eq. (30) from Eq. (29), we get the following equation from Eq. (33):

$$
\begin{aligned}
\sum_{n=-\infty}^{\infty}\{ & \frac{1}{2 \pi} \int_{-\pi}^{\pi}\left[c L^{\prime} H_{n-1}(\beta \mid c)(L(\theta) /|L(\theta)|)^{n-1}\right. \\
& \left.-\overline{c L^{\prime}} H_{n+1}(\beta \mid L(\theta))(L(\theta) /|L(\theta)|)^{n+1}\right] \\
& \exp (i z \theta) d \theta\} A_{n}=\frac{1}{2 \pi} \int_{-\pi}^{\pi} \frac{2}{\beta}\left[\frac{\left|L^{\prime}\right|}{\mu} f_{2}[\operatorname{Re} L(\theta),\right. \\
& \operatorname{ImL}(\theta)]-c \frac{\partial w^{(i)}[\operatorname{Re} L(\theta), \operatorname{Im} L(\theta)]}{\partial c} \\
& \left.-\bar{c} \frac{\partial w^{(i)}[\operatorname{Re} L(\theta), \operatorname{Im} L(\theta)]}{\partial \bar{c}}\right] \exp (-i z \theta) d \theta .
\end{aligned}
$$

\subsection{Conformal mapping for three cavity models}

In this paper, we design three cavity models, including a regular pentagon model (also called Model 1), a generalized quadrangular model (also called Model 2), and a horny model (also called Model 3). In $p$ domain, it is difficult to calculate scattering wave field of plane SH-waves by a certain model among the three models. Therefore, we adopt conformal mapping to simplify scattering wave field modeling, which is performed in $c$ domain.

\subsubsection{The conformal mapping for a regular pentagon model}

Figure 2 is the conformal mapping from an arbitrary polygon in $p$ domain to a unit circle in $c$ domain. The exterior angles of the arbitrary convex polygon are $\alpha_{1}, \alpha_{2}, \ldots, \alpha_{n}$ and the vertexes of the polygon are $p_{1}, p_{2}, \ldots, p_{n}$. The points $c_{1}, c_{2}, \ldots, c_{n}$ in $c$ domain located on the circumference of the unit circle correspond to $p_{1}, p_{2}, \ldots, p_{n}$ in $p$ domain, and $c_{k}=e^{i \eta_{k}}(k=$ $1,2, \ldots, n)$. On the basis of complex function theory, we have:

$$
p=F^{\prime}(c)=G \Pi_{k=1}^{n}\left(1-\frac{c_{k}}{c}\right)^{\alpha_{k} / \pi},
$$

where $G$ is a constant only related to the shape and direction of the polygon. By Eq. (35), an arbitrary

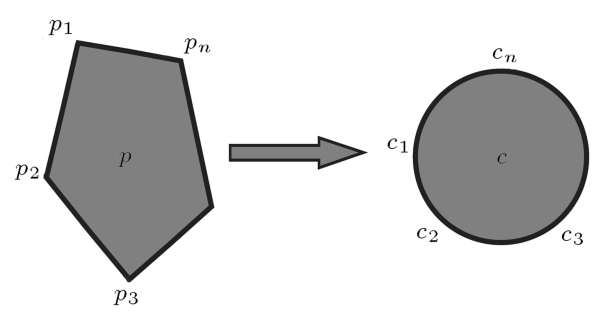

Figure 2. The conformal mapping from an arbitrary polygon to a unit circle. 
convex polygon in $p$ domain is transformed into a unit circle in $c$ domain.

According to the binomial theorem, Eq. (35) can be expanded to the following equation:

$$
p=F^{\prime}(c)=G\left[1-\frac{1}{\pi c} \sum_{k=1}^{n} \alpha_{k} c_{k}+O\left(\frac{1}{c^{2}}\right)\right] .
$$

The right side of Eq. (36) is zero if we take the value of variable $c$ on the vertexes. In this way, the character of the conformal mapping $p=F^{\prime}(c)$ is destroyed on these vertexes. To overcome this problem, we replace Eq. (36) by a polynomial corresponding with regard to $1 / \zeta$. On the condition of the polygon being equilateral, we denote the exterior angles as $\alpha_{k}=2 \pi / n(k=$ $1,2, \ldots, n)$. Furthermore, we assume that the $N$ points, $c_{1}, c_{2}, \ldots, c_{n}$, satisfy the equation $c^{n}-1=0$; thus, we have:

$$
F^{\prime}(c)=G\left(1-c^{-n}\right)^{\frac{2}{n}} .
$$

The derivation process of Eq. (37) is described in the Appendix.

For large $|c|$, Eq. (37) can be expanded as the summation of a power series with regard to $1 / c$. Thus:

$$
F^{\prime}(c)=G\left(1-\frac{2}{n c^{n}}+\frac{2(2-n)}{2 n^{2}} \cdot \frac{1}{c^{2 n}}+\ldots\right) .
$$

Integrating $F^{\prime}(c)$ and ignoring constant term, Eq. (38) becomes:

$$
\begin{aligned}
p= & F(c)=G\left[c+\frac{2}{n(n-1)} \cdot \frac{1}{c^{n-1}}-\frac{2(2-n)}{2 n^{2}(2 n-1)}\right. \\
& \left.\cdot \frac{1}{c^{2 n-1}}+\ldots\right] .
\end{aligned}
$$

Taking $n=5$ and $G=1$, and neglecting the high-order terms, we have:

$$
p=F(c)=c+\frac{1}{10 c^{4}}+\frac{1}{75 c^{9}} .
$$

Eq. (40) is a final expression for the conformal mapping from a regular pentagon to a unit circle (Figure 3 ).

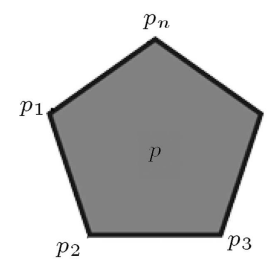

Physical domain

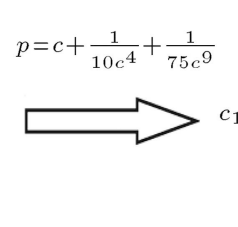

domain

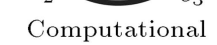

Figure 3. The conformal mapping from a regular pentagon to a unit circle.

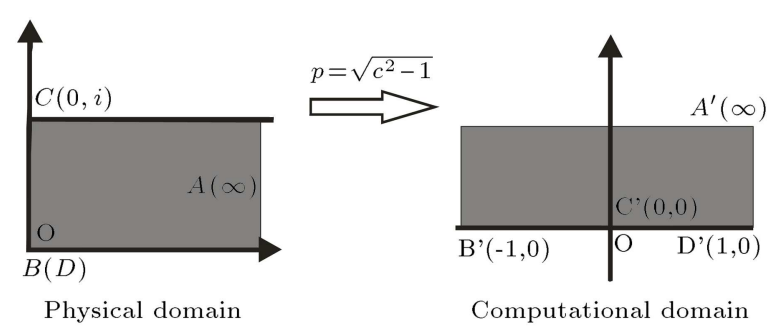

Figure 4. The conformal mapping from a general quadrilateral to an upper half plane.

\subsubsection{The conformal mapping for a generalized quadrangular model}

Figure 4 is the conformal mapping from a generalized quadrangular model in $p$ domain to an upper half plane in $c$ domain. The cross section of the cavity model in physical domain is marked as ABCD, where point A is located at infinite distance, and points B, C, and $\mathrm{D}$ are located at finite distance. Note that points $\mathrm{B}$ and $\mathrm{D}$ are located at the same position. If we calculate the wave field directly in physical domain, there will be singularity at the vortexes B, C, and D. According to the complex function theory, the problem will be successfully solved if we map the generalized quadrangular in $p$ domain to the upper half plane in $c$ domain. The corresponding conformal mapping is:

$$
\begin{aligned}
p & =p_{0}+K \int(c+1)^{-\theta_{B} / \pi} c^{-\theta_{C} / \pi}(c-1)^{-\theta_{D} / \pi} d c \\
& =p_{0}+K \sqrt{c^{2}-1}
\end{aligned}
$$

where $K$ and $p_{0}$ are two unknown constants. $\theta_{B}, \theta_{C}$, and $\theta_{D}$ are the exterior angles on the points $\mathrm{B}, \mathrm{C}$, and $\mathrm{D}$, respectively. According to point $\mathrm{B}$ and its corresponding point $\mathrm{B}^{\prime}$, we can get $0=p_{0}+A 0$; thus, $p_{0}=0$. According to point $\mathrm{C}$ and its corresponding point C', we can get $i=K i$, therefore, $K=1$. Thus, the conformal mapping is:

$$
p=\sqrt{c^{2}-1}
$$

\subsubsection{The conformal mapping for a horny model}

Figure 5 is the conformal mapping from a horny model in $p$ domain to an upper half plane in $c$ domain. The flare angle of the horny model is $\pi / 3$, the vertex is at the origin point, and angular bisector is along the real

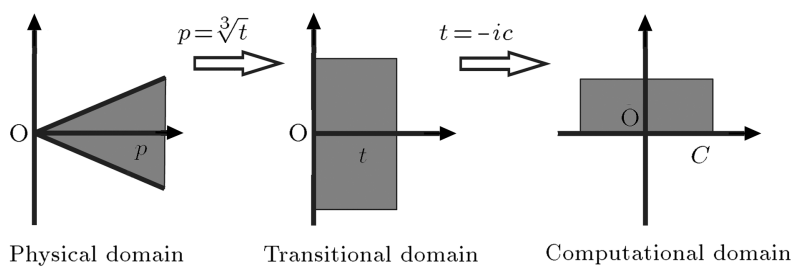

Figure 5. The conformal mapping from a horny model to an upper half plane. 
axis. That means:

$$
-\pi / 6<\arg p<\pi / 6 .
$$

For the model, we first transform the horny model in $p$ domain into right half plane in $t$ domain (or transitional domain) via conformal mapping $p=\sqrt[3]{t}$, and then transform the right half plane to upper half plane in $c$ domain through rotation transform $t=-i c$. In a word, we have the conformal mapping from a horny model to an upper half plane:

$$
p=-i \sqrt[3]{c}
$$

\section{Examples}

We consider plane SH-waves propagating on the $x_{1} x_{2}$ plane in this paper. The incident wave field is:

$$
w^{(i)}=b_{0} \exp [i \beta(p+\bar{p}) / 2],
$$

where $b_{0}$ is the amplitude, which is set as 1.5 in this paper.

We consider the displacement boundary condition for the first experiment. As to Model 1, the wave field on the boundary of the unit circle shows extreme value (Figure 6(a)), which verifies the wave field concentration principle. Note that it is hard to obtain the displacement wave field in $p$ domain because of the irreversibility of Eq. (41). For Model 2, the variations of the displacement wave field with position in real axis of Model 2 in $c$ domain are shown in Figure $6(\mathrm{~b})$. There are two peak values corresponding to the two singularities of the model in $p$ domain, namely $B(D)$ and $C$ (Figure 3 ). By introducing the inverse transformation of Eq. (42) into Eq. (30), we can get the scattering wave field in the $p$ domain (Figure 7(a)). For Model 3, we select 11 points on the boundary with the distribution of spatial coordinates from -5 to 5 and the interval as 1 . By substituting these points into Eq. (31), the displacement wave field on the boundary is obtained, shown in Figure 6(c). From the figure, we can observe only one extreme point corresponding to the angular point in $p$ domain. In addition, the displacement wave field on the boundary

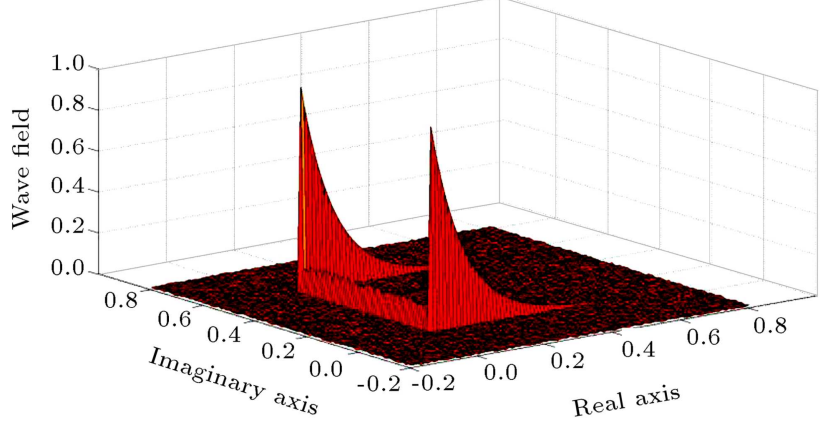

(a)

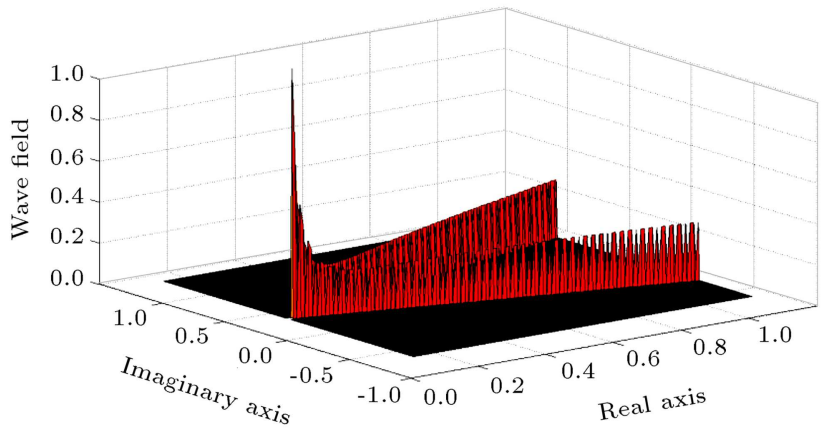

(b)

Figure 7. The displacement wave field maps in physical domain for (a) Model 2 and (b) Model 3.

and the angular point focus clearly. By substituting the inverse transformation of Eq. (42) into Eq. (30), we can get the scattering displacement wave field in $p$ domain (Figure $7(\mathrm{~b})$ ).

We consider the stress boundary condition for the second experiment. For Model 1, on the boundary of the unit circle, the stress wave field in $c$ domain also shows extreme value (Figure 8(a)), similar to the displacement wave field case (Figure 6(a)). However, curves for the stress wave field in Figure 8(a) are much rougher than those for the displacement wave field in Figure 6(a). For Model 2, the variations of the stress wave field with position in real axis of Model 2 in $c$ domain are shown in Figure 8(b). There are also two peak values corresponding to the two singularities of the model in $p$ domain, namely B (D) and C (Figure 3 ). By substituting the inverse transformation of Eq. (42)

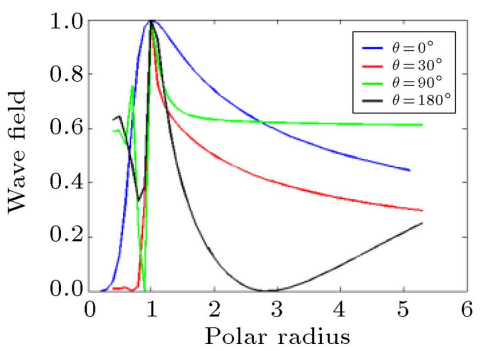

(a)

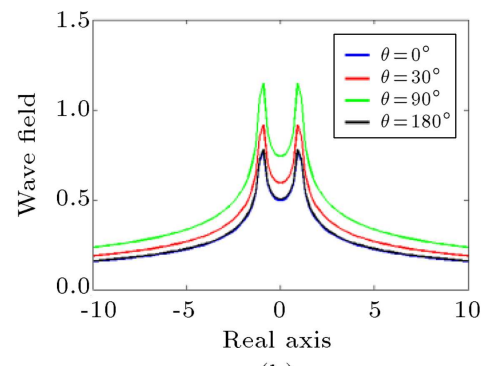

(b)

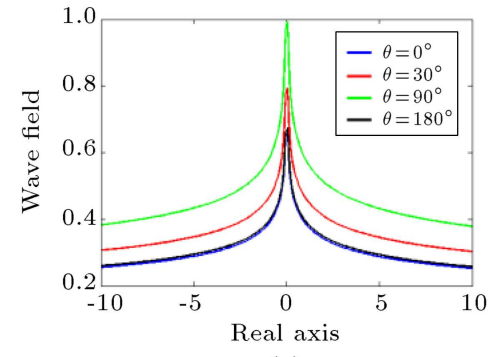

(c)

Figure 6. The displacement wave field curves with different incident angles in the computational domain for (a) Model 1, (b) Model 2, and (c) Model 3. 


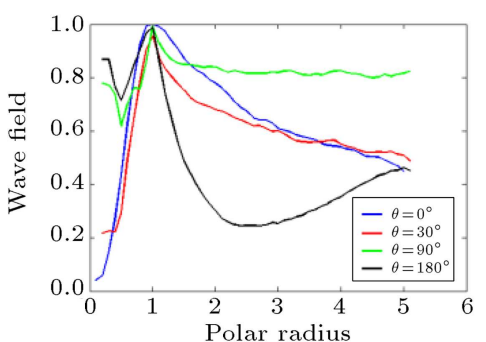

(a)

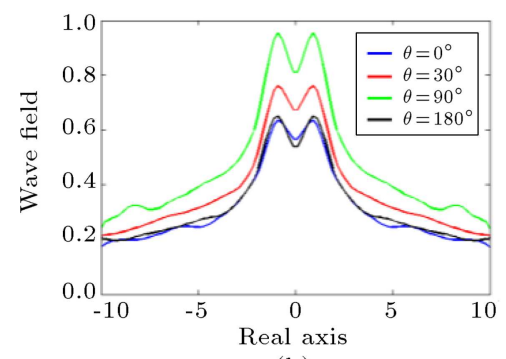

(b)

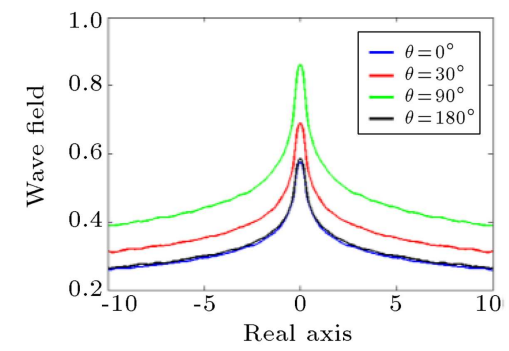

(c)

Figure 8. The stress wave field curves with different incident angles in the computational domain for (a) Model 1, (b) Model 2, and (c) Model 3.

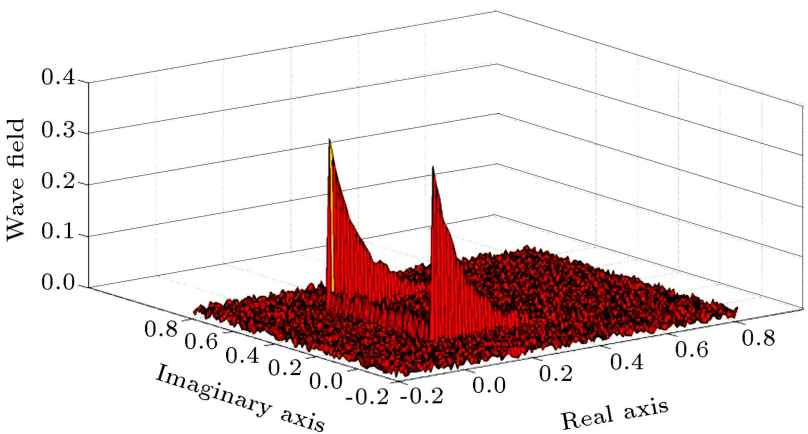

(a)

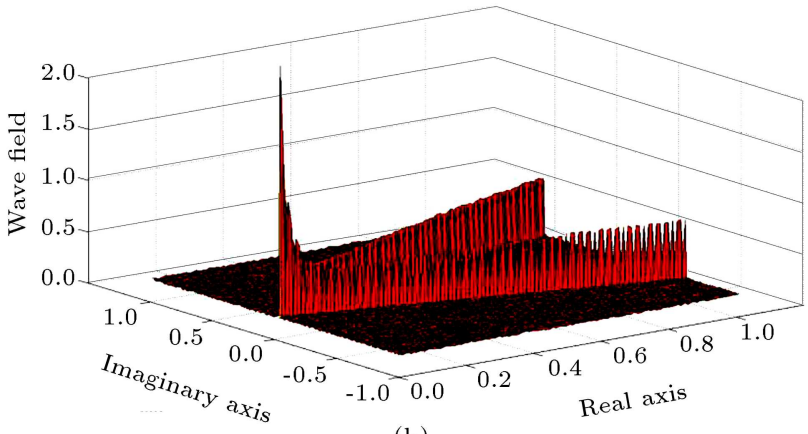

(b)

Figure 9. The stress wave field maps in the physical domain for (a) Model 2, and (b) Model 3.

into Eq. (34), we can get the scattering wave field in the $p$ domain (Figure 9(a)). For Model 3, we select 21 points on the boundary from -10 to 10 with step 1 . By substituting these points into Eq. (34), the stress wave field on the boundary is obtained, shown in Figure 8(c). From the figure, we can observe only one extreme point corresponding to the angular point in $p$ domain. In addition, the stress wave field on the boundary and the angular point focus clearly. By substituting the inverse transformation of Eq. (44) into Eq. (34), we can get the scattering stress wave field in $p$ domain (Figure 9(b)).

The two experiments by using either the displacement boundary condition or the stress boundary condition illustrate that the variation trends of the displacement wave field and those of the stress wave field are similar. In addition, they both accord with the wave field concentration principle.

\section{Conclusions}

By applying conformal mapping to the regular pentagon model, the generalized quadrangular model or the horny model, the wave field in the computational domain is analytically derived and numerically modelled with either a displacement boundary condition or a stress boundary condition. When the conformal mapping functions are reversible, the wave fields in the physical domain can also be obtained.

The two experiments illustrate that the wave fields for three different kinds of cavities mainly concentrate on the boundary of the corresponding cavity. It indicates a potential evidence of the existence of cavity, which is probably available information in the oil seismic exploration.

Although the conformal mapping method is applied to the scattering wave field model for three simplified cavity models with different features and shapes in this paper, it can also be used to deal with cavities with arbitrary shapes.

\section{Acknowledgements}

This research is partially supported by Sinopec Key Laboratory of Geophysics (Grant No. 33550006-15FW2099-0017).

\section{References}

1. Wang, S.X., Li, X.Y., Qian, Z.P., Di, B.R. and Wei, J.X. "Physical modelling studies of 3-D P-wave seismic for fracture detection", Geophysical Journal of the Royal Astronomical Society, 168(2), pp. 745-756 (2007).

2. Yuan, S.Y., Wang, S.X. and Tian, N. "Min Fresnel zone relative to large offsets", Oil Geophysical Prospecting (in Chinese), 44(4), pp. 387-392 (2009).

3. Gu, X.F. and Wang, Y.L. "Genus zero surface conformal mapping and its application to brain surface mapping", IEEE Transactions on Medical Imaging, 23(7), pp. 949-958 (2004).

4. Tarrass, I., Giraud, L. and Thore, P. "New curvilinear scheme for elastic wave propagation in presence of 
curved topography", Geophysical Prospecting, 59(5), pp. 889-906 (2011).

5. Yuan, S.Y., Wang, S.X., Sun, W.J., Miao, L.N. and Li, Z.H. "Perfectly matched layer on curvilinear grid for the second-order seismic acoustic wave equation", Exploration Geophysics, 45(2), pp. 94-104 (2012).

6. Yu, Y.C., Wang, S.X., Yuan, S.Y. and Qi, P.F. "Phase estimation in bispectral domain based on conformal mapping and applications in seismic wavelet estimation", Applied Geophysics, 8(1), pp. 36-47 (2011).

7. Herrmann, F.J., Friedlander, M.P. and Yilmaz, O. "Fighting the curse of dimensionality: compressive sensing in exploration seismology", IEEE Signal Processing Magazine, 29(3), pp. 88-100 (2012).

8. Yuan, S.Y., Wang, S.X., Luo, C.M. and He, Y.X. "Simultaneous multitrace impedance inversion with transform-domain sparsity promotion", Geophysics, 80(2), pp. R71-R80 (2015).

9. Eduardo, H.M. and Jose P.C. "Asymptotically almost periodic and almost periodic solutions for a class of partial integrodifferential equations", Electronic Journal of Differential Equations, 2006(38), pp. 1-8 (2006).

\section{Appendix}

From Eq. (36), we have:

$$
F^{\prime}(c)=G \Pi_{k=1}^{n}\left(1-\frac{c_{k}}{c}\right)^{\frac{2}{n}}=\frac{G}{c^{2}} \Pi_{k=1}^{n}\left(c-c_{k}\right)^{\frac{2}{n}} .
$$

If each $c_{1}, c_{2}, \ldots, c_{n}$ satisfies $c^{n}-1=0$, then:

$$
\Pi_{k=1}^{n}\left(c-c_{k}\right)=c^{n}-1 .
$$

By substituting Eq. (A.2) into Eq. (A.1), Eq. (37) can be obtained:

$$
\begin{aligned}
F^{\prime}(c) & =\frac{G}{c^{2}} \Pi_{k=1}^{n}\left(c-c_{k}\right)^{\frac{2}{n}} \\
& =\frac{G}{c^{2}}\left(c^{n}-1\right)^{\frac{2}{n}} \\
& =G\left(1-c^{-n}\right)^{\frac{2}{n}} .
\end{aligned}
$$

\section{Biographies}

Wei Chen, a Doctor at China University of Petroleum-Beijing, was born in December 1985. His research interests include seismic wave field analysis of heterogeneous media, seismic physical simulation experiment, and seismic data imaging.

Shangxu Wang, a Professor at China University of Petroleum-Beijing, was born in December 1962. He is currently a Doctoral Supervisor. His research interests include seismic physical simulation experiment, seismic imaging, and seismic data imaging. Professor Wang has made outstanding achievements in the field of exploration geophysics. 